\title{
Equipoising Multi-modal Traffic Environments using Vehicular Ad-hoc Networks
}

\author{
S.T. Rakkesh, A.R. Weerasinghe, R.A.C. Ranasinghe
}

\begin{abstract}
Competent transportation infrastructure is one of the prominent factors influencing sustainable economic growth and appreciable living standard in a country. Urban cities habitually equipped with multi-modal traffic flows which encompass various modes of transportation mechanisms such as walking, skating, cycling, motorcycles, three wheelers, private passenger cars, public transport modes like buses or trains, etc. Efficient urban traffic planning has become an exigent task involving complex behavioural analysis of multi-modal traffic scenarios. One possible way of enhancing the travel experience of commuters is by revolutionizing the vehicular communication strategies to devise intelligent routing approaches. Vehicular adhoc networks (VANETs) are a special type of mobile ad-hoc networks (MANETs) which can be used to define intelligent conciliator interfaces for vehicle to vehicle (V2V) or vehicle to infrastructure (V2I) communication needs. In this paper, we propose an approach for equilibrating multi-modal traffic environments after road block situations occur through VANET scenario simulations. For this research, we have used OMNeT++ network simulator to simulate the mobile network involved and SUMO microscopic traffic simulator to simulate vehicular movements in parallel and Veins framework as a bridging interface between OMNeT++ and SUMO. We have validated our solution with two heterogeneous metropolitan areas located in the cities of Kandy and Colombo in Sri Lanka. Our solution achieved lower values for waiting time and trip duration of vehicles and qualitatively improving the traffic flow when comparing against the absence of equilibrating module.
\end{abstract}

Keywords - microscopic; VANET; multi-modal; simulation

\section{INTRODUCTION}

Along with the growth of human settlements across the world, metropolitan areas and traffic congestion have developed concomitantly. The same causes that draw people to congregate in large urban cities also lead to sometimes unendurable levels of traffic congestion on urban streets and surrounding areas. New endeavours of research solutions and effective traffic plans should be explored and implemented to ease congestion and improve traffic flow over the regions. Traditional transportation planning methodologies were formulated during an era where urban traffic environments were growing rapidly from preliminary stages so transport planning focused primarily on infrastructure expansion.

Manuscript received on $1^{\text {st }}$ December, 2016. Recommended by Dr.T.M.H.A.Usoof on $24^{\text {th }}$ November, 2017. This paper is an extended version of the paper, "A Decentralized Vehicle Re-routing Approach using Vehicular Ad-hoc Networks" presented on ICTer2016 Conference.

Titus Rakkesh is reading for $\mathrm{PhD}$ in Artificial Intelligence area from University of Colombo School of Computing (UCSC). He is currently working as an expert in software engineering \& implementation at Dialog Axiata PLC. (titus.rakkesh@dialog.lk)

Dr. Ruvan Weerasinghe is a senior lecturer at the University of Colombo School of Computing. (arw@ucsc.cmb.ac.lk).

Dr. R.A.C. Ranasinghe is the Chief Executive Officer at IdeaHub (Pvt) Ltd. (chaminda@ideahub.lk)
However, economic constraints and territorial limitations have proved to be too formidable for continuing the roadway expansions. Present day urban areas are experiencing an incredible expansion in terms of both population and financial power of their inhabitants. This financial liberty allows more and more citizens to have their own private vehicles and to take the comfort and luxury of mobility and communication for granted. Current traditional transportation planning strategies attempt to regularize vehicular speeds, decrease traffic congestion and minimize accident rates using well structured limited set of engineering, architectural, legislative and financing tools. Conventional transportation analysis tools tend to focus on a limited set of impacts, hence the effects caused by the current multi-modal traffic environments tend to be unnoticed by the traditional transportation framework, since they are relatively too much convoluted to quantify or analyse with the current traffic management strategies involved.

Intelligent transportation systems (ITS) [1] are playing a crucial role on providing excellent solutions for complex traffic problems since last decade. Advances in ubiquitous computing [2] and wireless technologies [3] have offered new trends in intelligent transportation systems, which aim at providing break through solutions enhancing travel experience and improving safety. In recent years, transportation planning has expanded to include more emphasis on non-vehicular requirements and more concern in factors such as safety of pedestrians, health impacts and environmental effects. Researchers are more expected to adopt multi-disciplinary solution approaches, especially due to the rising environmental and socialistic concerns. Thus, current research trends in the field of transportation planning were stirred more towards multi-modal and comprehensive traffic analysis taking into account of wider range of options and impacts.

Multi-modal transportation planning is complex because transport modes differ in various aspects including their availability, speed, popularity, costs, constraints and usability. Traffic analysis becomes even more complex because each mode includes various subcategories with distinctive characteristics. If we take "pedestrian" transport mode as an example, this include people walking alone and in groups, people standing, joggers, people walking with pets, blind people who are using special canes, people who are carrying goods, people who are running, etc. Their actual requirements, skills, impacts and interactions with the traffic environment can vary radically. Multi-modal transport planning requires strategies for evaluating the effect of each mode on the traffic environment and this diversity among the modes make the combined traffic planning a significantly complex problem.

As an emerging new form of wireless networks, vehicular ad-hoc networks (VANETs) [4] have become the trend setter 
in intelligent transportation systems. VANETs support a large spectrum of decentralized vehicular applications ranging from traffic light optimization solutions to dynamic vehicle rerouting applications. VANET solutions are a type of solutions built from the concept of establishing an interconnected spontaneous network of vehicles to fulfil a traffic requirement or handle a scenario. Typically, solutions which uses VANETs design blueprint target to build a distributed inter vehicular communication (IVC) [5] network which is autonomous, cost efficient and based on flexible architectural design principles. Simulators play a significant role in evaluating newly developed applications which use VANETs, since directly carrying out tests for solutions in real traffic environments is not feasible usually as it require extensive infrastructure changes and substantial support staff engagement. Both vehicular traffic simulations and network layer simulations should be jointly performed in order to evaluate the effectiveness of inter vehicular communications (IVC) on vehicle routing strategies, congestion resolution, safety measures, travel experience, etc and to optimize the solutions. Vehicular traffic simulators generate required realistic vehicular mobility demand to be used in network simulators as an input. Network simulators map these vehicles as nodes in a wireless network like structure and simulate data dissemination topologies between nodes by emulating data transfer. Since traffic simulators and network simulators differ significantly in structure, usually there is a concrete requirement for a mediation framework operating between them.

In this paper, we propose an intelligent solution approach to equilibrate multi-modal traffic environments when road blocks or accidents occur. For our study we have selected SUMO [6] as the traffic simulator, OMNeT++ [7] as the network simulator and Veins toolkit [8] as the mediation framework between SUMO and OMNetT++. Fig 1 describes few popular traffic simulators, network simulators and mediation frameworks and the toolset we selected for our study, where as fig 2 illustrates more detailed overview of the selected toolset for our experiment.

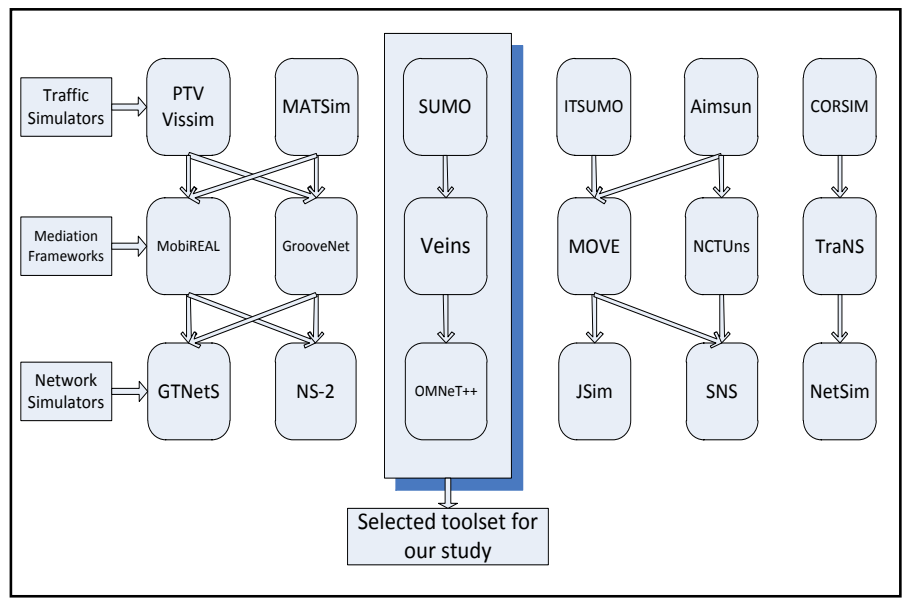

Fig 1. Overview of various simulators and some sample interaction flows

Section IV describes the conditions and constraints of the experiments. Section V presents the analysis and discussion of output results obtained.

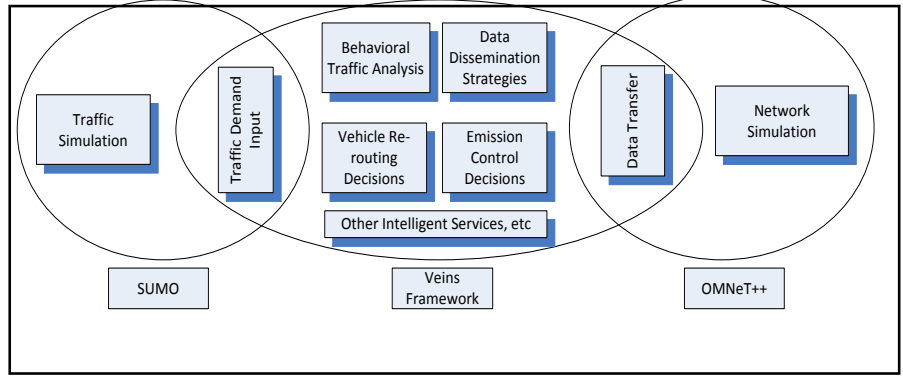

Fig 2. More detailed overview of selected toolset for the study

In section VI, we discuss the related research works from the literature with brief comparison with our study. Conclusions and future work are given in Section VII.

\section{BACKROUND STUDY}

In this section, we have focused on explaining the basic concepts and brief overview of multi-modal mobility patterns, VANETs and the components of the tool set we have used for our research study. We feel presenting this information is worthwhile, before moving into the experiment details and results analysis.

\section{A. Multi-modal Vehicular Traffic}

Multi-modal traffic modes can be broadly classified into private transportation modes and public transit avenues. Private transport is a transportation mode which is not available to be used by the general public in a shared manner. Private transport is the leading form of transportation around the world due to the fact that it is more convenient for the traveller. Private transport mode can be further categorized into motorized and non-motorized forms. Motorized option includes motor cycles, three wheelers, passenger cars, private boats, private jets, delivery vehicles, etc and non-motorized option includes pedestrian movements, skaters, bicycles, garden carts, hot air balloons, etc. These sub categories can be further divided into sub divisions. If we take "passenger automobiles" sub division as an example, this can include sedans, saloons, hatchbacks, station wagons, limousines, mini vans, vans, single cabs, double cabs, jeeps, SUVs, etc. These vehicles extensively vary from each other in their length, shape, acceleration ability, braking capability, speed, turning capacity, etc. This significant diversity among vehicles poses a challenge in multi-modal transport planning when enforcing speed limit restrictions, defining parking slots, provisioning traffic light cycle arrangements, pedestrian crossings placement, defining emission control strategies, enforcing safety and security rules and infrastructure expansion proposals. Hence determining the cumulative effect of divergent vehicles on a traffic environment is not a trivial task.

Public transport is a shared transportation mode which is available for use by the general public. Public transportation varies significantly from private transportation from financing, convenience and availability perspectives. Public transportation is mostly funded by governments, while private transportation is established and financed through private business ventures and individuals. Public transport is generally regarded as significantly more energy efficient and greener, cheaper, in some selective occurrences convenient and quicker and causing less parking issues than other private transportation options. Public transport mode includes shared taxis, vans, buses, trains, coaches, ferries, ships, airlines, etc. These avenues differ drastically among themselves and 
modes like trains, ships and airlines require dedicated infrastructure arrangements and specialized support staff networks. Most of the public transportation modes are administrated by the policy makers are usually organized to serve the general public better and passenger fares are heavily subsidized. Hence its services may have been constrained by the amount of revenue it receives. Due to these limitations, public transportation may operate only during certain hours and days in selected regions and there may be some areas in which public transportation facilities are not available at all. For commuters, selecting between private and public transportation modes often involves considering availability, handiness, affordability, return trip plans and the safety of each option. In many cases, commuters may find that utilizing a combination of public transit and private transport options is a better preference and makes the most sense. Thus, modelling the optimum inter-dependency travel options between private and public transportation modes is one of the vital challenges of efficient multi-modal traffic analysis process.

\section{B. VANET}

Vehicular ad-hoc networks (VANETs) are a distinct type of mobile ad-hoc networks which are mainly used in building intelligent transportation systems (ITS). VANETs primarily focus on defining communication topologies for vehicle to vehicle (V2V) [9] data transfer and vehicle to infrastructure (V2I) [10] communication technologies. Implementing high speed short range communication channel interfaces with minimal connection failure is one of the crucial challenges faced when designing an effective VANET scenario. Since last decade, several research investments have done from both individual researchers and automobile industry on finding new enhanced communication protocols and interfaces suitable for vehicular networks.

The increased number of casualties and injuries caused by vehicle accidents each year, places a definite and urgent requirement for effective road safety applications and devices to be build. Thus building road safety scenarios have become one of the crucial applications of VANETs among its other use cases ranging from enhancing travel experiences to providing better infotainment applications. In VANET, when a non-safe situation is detected, the warning messages are usually propagated to the vehicles in the neighbourhood. The neighbourhood of a vehicle is defined by the VANET design and the traffic entities belong to a neighbourhood is determined by various parameters, such as proximity, vicinity, velocity, impacts on other entities, etc. Beaconing [11] is widely used in VANET scenarios to discover and sustain the neighbourhood relationships. Even though VANET solutions primarily focus more on improvised wireless networks, usage of standard cellular networks and conventional road side units (RSUs) [12] can play a crucial supporting role when designing and operating VANET solutions in real urban traffic environments. Fig 3 depicts various communication interfaces which can be possibly used in a VANET scenario.

Recent innovations in wireless technologies, such as enhancements in dedicated short range communication (DSRC) [14] and improvements in long term evolution (LTE) [15] paved new ways of communication channels for inter vehicular communications (IVC). Since VANETs are based on flexible architectural design principles, solutions can use any combinations of wireless technologies, such as ZigBee,

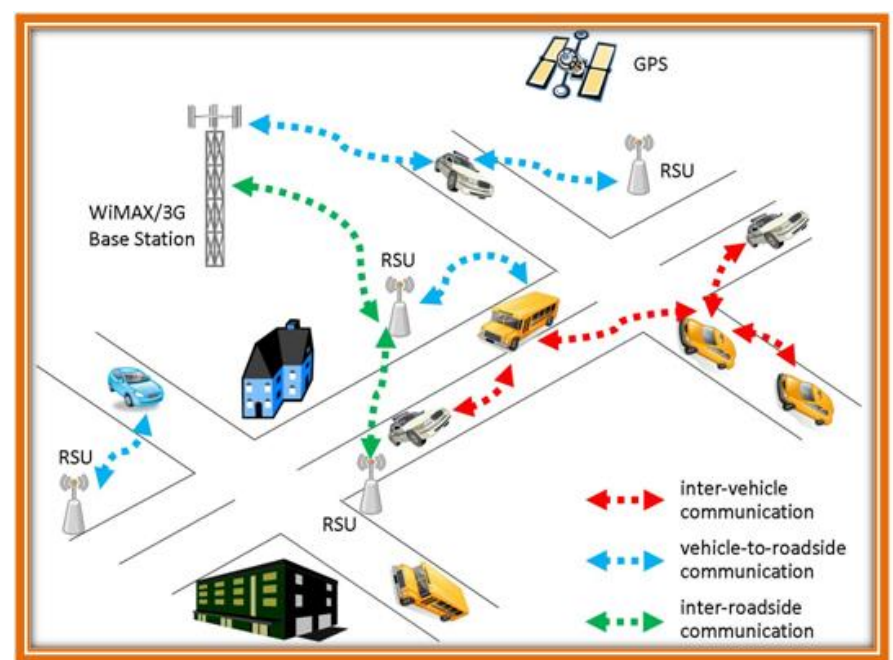

Fig 3. Various communication interfaces in a VANET scenario [13]

DSRC, Infrared, Bluetooth, WiFi, etc and standard cellular technologies like GSM, UMTS, LTE, WiMAX, etc. Also VANET solutions have the freedom to choose any blend of data propagation techniques, such as unicasting, multicasting or broadcasting for transmitting messages between the traffic entities.

\section{OMNeT++}

Evaluating the efficiency and reliability of new protocols for wireless networks or validating the newly proposed networking solutions is vital before implementing them in the real world. Network simulation is widely used for these evaluation purposes, since this methodology is cost efficient and eliminates the complexity involved in actual deployments. OMNeT++ gained widespread popularity as a discrete object oriented simulation library and framework, largely used for building complex network simulations. Although OMNeT++ itself not a simulator comprising concrete implementation, it is comprehensively equipped with well structured interfaces and tools which can be used for writing viable simulations. In this paper we have used OMNeT++ to build the network simulation portion of our experiment.

\section{SUMO}

Vehicular mobility simulations play a significant role in evaluating and validating traffic solutions before deploying them in real urban traffic environments. Simulation of Urban MObility (SUMO) is a well-known open source traffic simulator that can be used as a highly portable and microscopic road traffic simulation tool which is skilful in handling large traffic scenarios. SUMO can hold the information about the study region in a "network" file using nodes, edges, and connections between them. This network file can be generated by importing a map from popular digital mapping software like OpenStreetMap [16] or traffic management software like MATSim [17] and converted to a valid SUMO network. The "routes" file in SUMO is the traffic demand definition file which contains the information of all the streets and lanes each individual vehicle will pass through as route definitions. SUMO provides the feature of extensibility by providing means of adding "additional" files. These additional files can be used to define traffic light cycles, define bus stops or to generate various output files containing different statistical information. SUMO has an extended API interface called "Traffic Control Interface (TraCI) [18]" to 
manipulate vehicular mobility parameters during the runtime. In this experiment TraCI is used as an API interface to connect SUMO with other components.

\section{E. Veins Framework}

In order to build a proper VANET solution, the network simulation component and the traffic simulation component should be loosely coupled with an interface which can act as a mediator between two simulators to pass control messages. As depicted in Fig 1 several combinations of frameworks and simulators can be used to build a viable VANET solution. In this study, we have chosen Veins framework as a bridging interface to couple SUMO and OMNeT++. Veins framework is an open source simulator interface for running vehicular network simulations, which supports dynamic rerouting of vehicles in response to the network simulator's feedbacks, adheres with IEEE 802.11p and IEEE 1609.4 DSRC/WAVE network layers standards, comprising features for modelling standard cellular networking protocols, and supports the operations for multi-channel data transfer, quality of service (QoS) channel access, noise and interference effects. For our experiments, we are running SUMO and OMNeT++ in parallel connected via Veins framework using TCP sockets.

\section{EXPERIMENTAL SETUP}

In this section we present the experimental setup we followed to formulate our VANET study scenario and how the output results were generated from the experiments. First, we describe how the study scenario was prepared in the simulation environment specifically for this research. Later we explain the implementation details and input parameters for our experiment and in the subsequent sections we suggest the conclusions which can be derived based on the analysis of the output results.

\section{A. Research Scenario}

We have taken portions from Colombo and Kandy cities in Sri Lanka as study regions for our experiment. These are among bespoken metropolitan areas comprising heavy traffic flows usually during the peak hours. We have used OpenStreetMap, a well-known open source digital mapping software and extracted the geographical information of both cities including places of point of interest (POI), roads, lanes, intersections, highways, roundabouts, bridges, railway lines, water channels, traffic lights, etc and converted to SUMO network. We have selected two scenarios for our experiment, since they compose quite different urban areas with structural dissimilarities and heterogeneous traffic organization. We have formulated the solution model for this research study based on simulation approach of diverse types of transportation forms. We have introduced different types of vehicles to make the experiment comprise multi-modal and heterogeneous mobility patterns. The initial traffic demand was generated using synthetic traffic demand generation process by assigning random source and destination points for vehicles and the traffic lights cycle logic and phase durations were provisioned using SUMO's static traffic light cycle generator module. In this way, the preliminary setups for research scenarios in SUMO were prepared. Then using the Veins framework, traffic nodes (vehicles) simulated in SUMO traffic simulator are mapped with network elements depicted in OMNeT++ network simulator. Periodic accident situations were generated via Veins framework and the broadcasting of notification messages to the neighbourhood vehicles are jointly carried out by Veins framework and OMNeT++ simulator, while both SUMO and OMNeT++ simulators are running in parallel coupled by the Veins framework. In this paper, we have validated the efficiency of a rerouting approach using both our study regions from Colombo and Kandy by comparing few specific result parameters with and without the rerouting approach enabled. In the subsequent section, we will describe the implementation detail in depth. Table 1 depicts the number of simulated vehicles moving around during the simulation of both scenarios and other input parameters. And Table 2 depicts different vehicle types within the traffic demand with their main characteristics which were moving around during the simulation. In Fig. 4, the selected areas of our study regions are shown with their capture views in Google Earth, OpenStreetMap and SUMO.

TABLE I.

COUNT OF MOVING VEHICLES DURING THE SIMULATIONS

\begin{tabular}{|c|c|c|c|}
\hline Scenario & Research Area & $\begin{array}{l}\text { Number of } \\
\text { Vehicles }\end{array}$ & $\begin{array}{c}\text { Simulation } \\
\text { Time (Steps) }\end{array}$ \\
\hline \multirow{2}{*}{$\begin{array}{c}\text { With } \\
\text { rerouting } \\
\text { enabled }\end{array}$} & $\begin{array}{c}\text { Colombo } \\
\text { Region }\end{array}$ & 50000 & 3000 \\
\hline & $\begin{array}{l}\text { Kandy } \\
\text { Region }\end{array}$ & 50000 & 3000 \\
\hline \multirow{2}{*}{$\begin{array}{c}\text { Without } \\
\text { rerouting } \\
\text { enabled }\end{array}$} & $\begin{array}{c}\text { Colombo } \\
\text { Region }\end{array}$ & 50000 & 3000 \\
\hline & $\begin{array}{l}\text { Kandy } \\
\text { Region }\end{array}$ & 50000 & 3000 \\
\hline
\end{tabular}

TABLE II.

COUNT OF MOVING ENTITIES DURING THE SIMULATIONS

\begin{tabular}{|c|c|c|c|}
\hline Vehicle Type & $\begin{array}{c}\text { Length(netto } \\
\text { length unit) }\end{array}$ & $\begin{array}{c}\text { Max } \\
\text { Speed(m/sec) }\end{array}$ & $\begin{array}{c}\text { Total Count } \\
\text { in Simulation }\end{array}$ \\
\hline Bicycle & 0.5 & 15 & 250 \\
\hline Motor Cycle & 0.5 & 40 & 1000 \\
\hline Three wheeler & 0.5 & 40 & 20000 \\
\hline Sedan Car & 4.5 & 60 & 10000 \\
\hline Hatchback Car & 4.0 & 60 & 10000 \\
\hline Station Wagon & 5.25 & 60 & 2000 \\
\hline Van & 5.50 & 60 & 3000 \\
\hline Truck & 8.0 & 50 & 1000 \\
\hline Delivery Vehicle & 6.0 & 50 & 1000 \\
\hline Bus & 10 & 50 & 1750 \\
\hline
\end{tabular}

\section{B. Implementation Details}

In this study, we have some conditions and assumptions presumed for the experiments. We assume that all vehicles are capable of communicating with each other through wireless networking protocols and having global positioning system (GPS) device installed. This GPS device is assumed to have the information of each vehicles location, the information about the lane in which the vehicle is travelling, such as length of the lane, maximum speed limit allowed in that lane, etc and the knowledge of the overall map of the area the vehicle is intending to pass-through. We have also assumed that each vehicle's route has been pre-determined during the simulations and the rerouting decision making component is deployed in each vehicle.

We have formulated the solution model for this research study In order to analyze the combined effect of public transport and private transport, based on simulation approach 


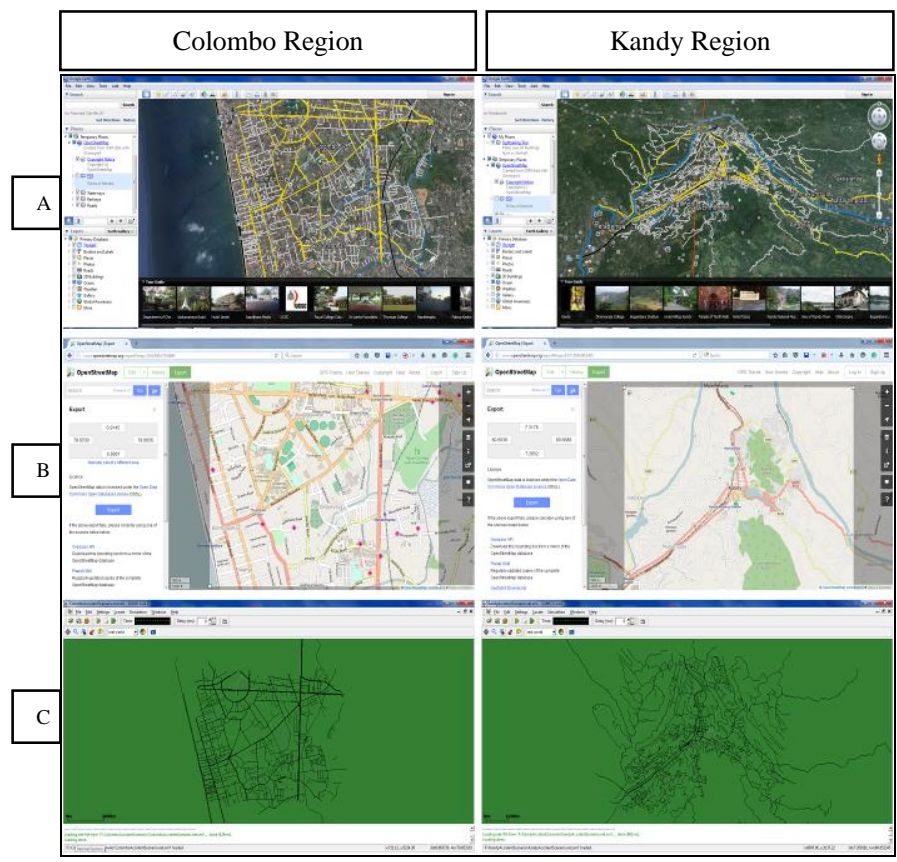

Fig.4. Preparation of study regions for research - Colombo, Sri Lanka and Kandy, Sri Lanka. Selected the area of interest from Google Earth (A) and extracted the selected boundary area from the OpenStreetMap (B) and then generated SUMO network (C) for simulation purposes.

of diverse types of transportation forms. We have introduced different types of vehicles to make the experiment heterogeneous. In order to analyze the combined effect of public transport and private transport, we have defined bus halts with explicitly set stopping durations and modeled bus traffic demands. In our simulations, vehicular traffic flow was managed by SUMO, while scheduling, executing and resolving accidents is jointly handled by Veins framework and $\mathrm{OMNeT}++$ simulator. For evaluation purposes, using Veins framework, we are periodically scheduling accidents with the rate of one accident per 30 seconds randomly over the region and assess the efficiency of the rerouting approach by enabling and disabling the rerouting component. When an accident is simulated the vehicle which was involved broadcasts the accident message along with its location details to the vehicles in the neighbourhood. The neighbouring vehicles which receive the accident messages have to act based on their location, accident location and their intended travel path. Based on these criteria, some vehicles can safely ignore the messages since their intended travel path may not be affected by the occurred accident. Some vehicles might be in a position not able to apply the rerouting logic due to their location and the accident location. These vehicles need to slow down or stop until the accident vehicle gets removed in the simulation. Other category of neighbourhood vehicles is the one which can change their routes according to the rerouting approach logic to avoid the traffic congestion created by the accident. While fig. 5 shows a parade of heterogeneous vehicles for a demonstration purpose, fig. 6 depicts different types of vehicles moving around two adjacent roundabouts near Ward Place during the Colombo scenario. Fig. 7 depicts a bus halt where buses stop to pick up passengers during the simulation. Fig 8 depicts how a node turns into red when it is getting stopped in the simulation due to an accident and how the accident related messages are propagated between the nodes. And fig 9 illustrates how neighbourhood nodes were determined for a particular node in the network and how message dissemination takes place between neighbouring nodes during our Colombo scenario.

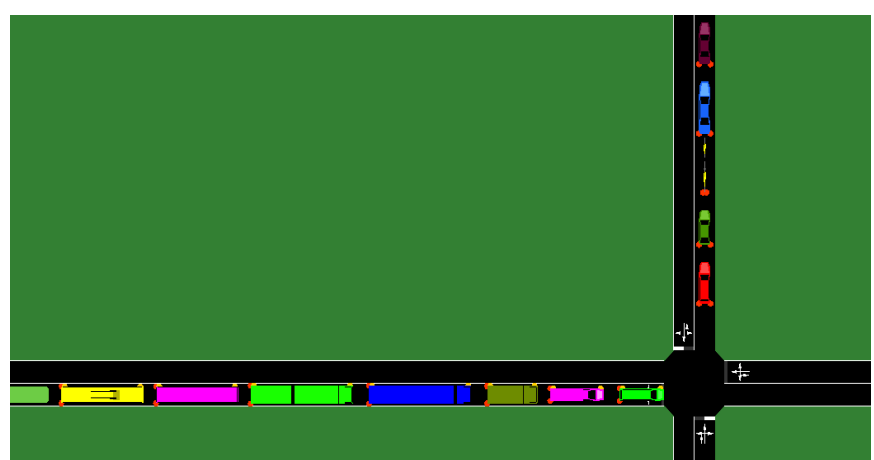

Fig 5. Parade of heterogeneous vehicles to demonstrate the multi-modal vehicles used in the study scenarios.

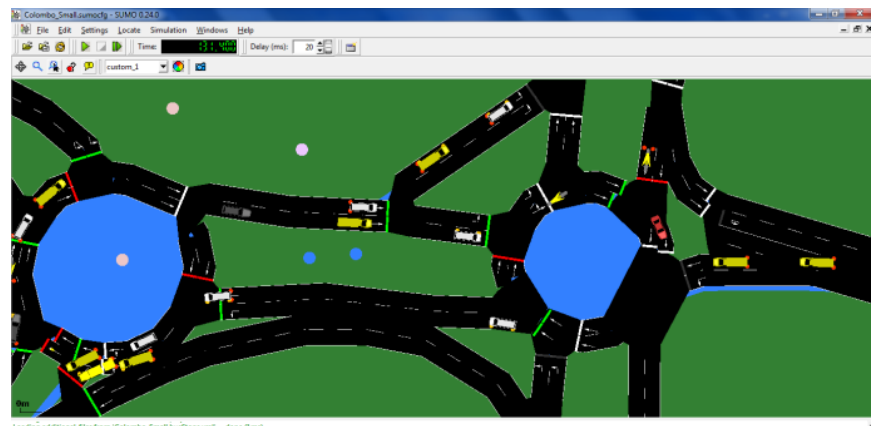

Fig.6. Depicts heterogeneous vehicles moving near Ward Place in Colombo Scenario, Sri Lanka

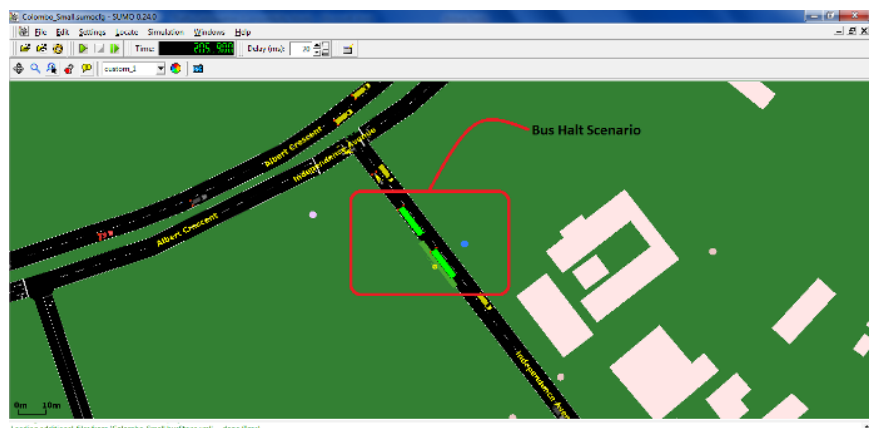

Fig 7. Depicts a bus halt near Independence Avenue, where a another bus slowing down to stop during Colombo scenario.

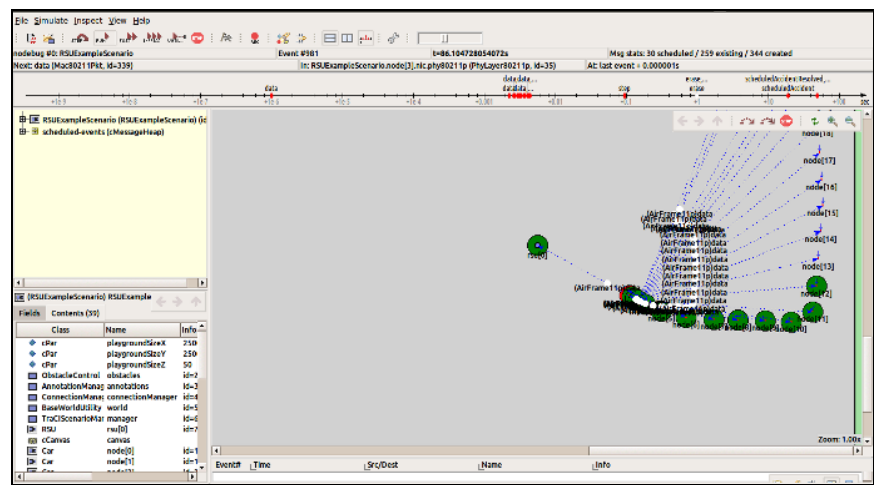

Fig 8. Depicts how a node turns into red after an accident and how the accident related messages are propagated between the nodes.

In reality the vehicles involved in an accident have to be removed from the accident location by either driving the 


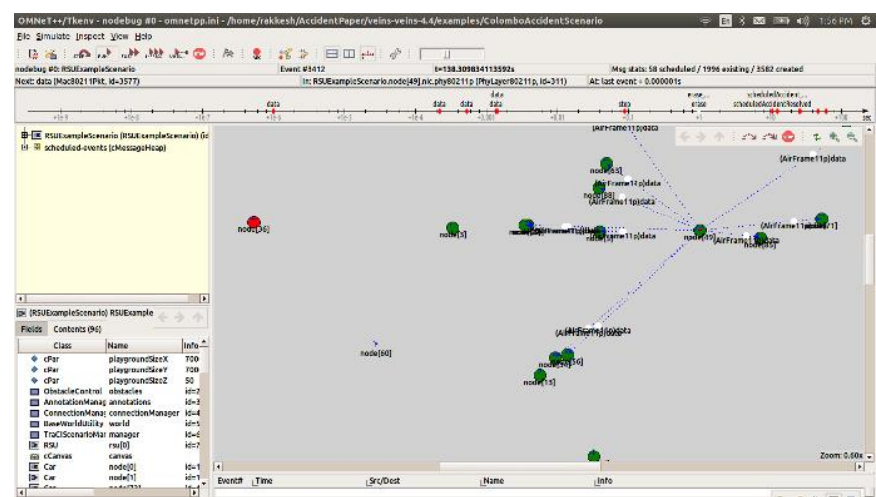

Fig 9. Depicts the neighbouring nodes selection and message broadcasting techniques displayed during Colombo study scenario.

vehicle by itself or towing by another vehicle. So in our experiments, we are simulating the removal and communicating this as special release messages to other vehicles by broadcasting. When the vehicles receive this release message they act based on their location, accident location and their planned travel path. For the vehicles where the accident is not in their route or trajectory, they can safely ignore these messages. There can be some other vehicles which can be directly affected by the accident and they can be completely stuck in the traffic. With this release message these may start to move or increase their speeds. And there is another group of vehicles which is not located on the path where accident occurred directly, but their intended path contains the lane in which the accident has taken place. When they receive the release messages, these vehicles can revalidate their best possible paths to their destinations based on the updated information and do rerouting if necessary.

\section{SIMULATION ASSUMPTIONS}

In this section, we explain the constraints and conditions we pursued during our experiments. The following are the important conditions and constraints which were entangled during our simulations.

- The vehicular traffic demand simulated during the experiments was synthetic data generated using random source and destination points. Table 1 and table 2 demonstrate the input parameters used during experiments for each simulated scenarios.

- Pedestrian movements, railway traffic operations, vehicle parking measures and shipping canal travel procedures were not considered during our scenarios.

- Predefined traffic light cycles and fixed phase durations were used during the simulations which were generated with static traffic light cycle generating features shipped with SUMO.

- During the experiments, accidents were simulated with the rate of one accident per 30 seconds randomly over the region to evaluate our proposed rerouting strategy. The possible scenarios of increasing and decreasing the accident rate were not considered during the simulations.

- During the simulations, the vehicles which were involved in an accident were removed after a predefined duration. But the scenarios of keeping the accident vehicles in the network permanently or removing with varying intervals were not simulated during the trials.
While fig 10 illustrates speed limit restrictions enforced during the simulations, fig 11 demonstrates traffic light locations. The location of the traffic lights and the speed restrictions enforced are predefined for the study scenarios. In Kandy scenario, the selected study area does not contain any traffic lights.

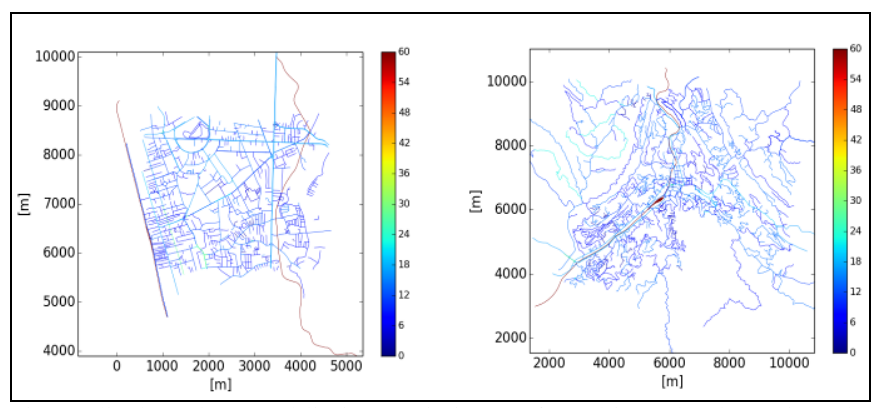

Fig 10 displays the speed limits restrictions enforced in Colombo, Sri Lanka and Kandy, Sri Lanka study regions respectively.

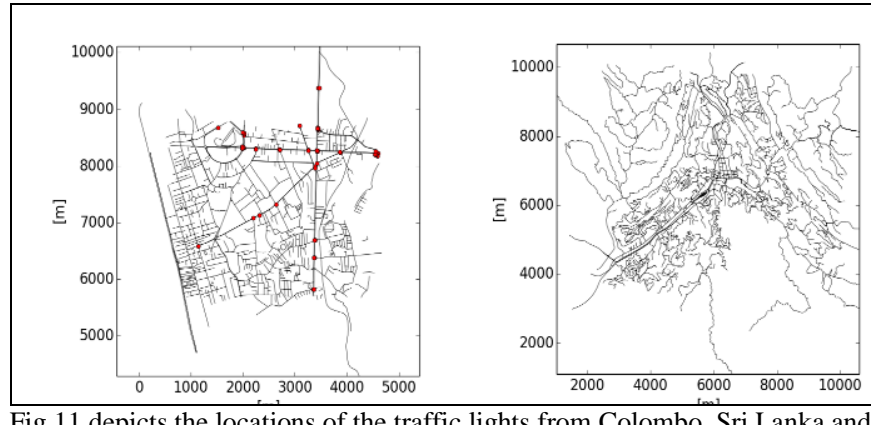

Fig 11 depicts the locations of the traffic lights from Colombo, Sri Lanka and Kandy, Sri Lanka study regions respectively.

\section{ANALYSIS AND DISCUSSION OF RESULTS}

In this section, we present the summarized results we obtained from our experiments and the conclusions we can propose based on the results. Also, we explain the analyses process we followed to filter useful information from the generated output during the simulations. For analysis purpose, we have selected the trip duration and waiting time of vehicles for the completed journeys during the experiment window of 3000 simulation steps as comparison metrics. The following are the brief description of these comparison attributes.

- $\quad$ Trip duration - The time the vehicle took to reach its destination traversing all the lanes and intersections in its intended path (Measured in simulation step units)

- Waiting Time - The number of micro step units in which the vehicle's velocity was below $0.1 \mathrm{~m} / \mathrm{s}$. (In our simulation, the number of total micro step units are 30000, which is derived by dividing each simulation step unit by 10.)

Fig 12 illustrates the summarized trip duration we have obtained when both the rerouting approach was enabled and disabled during Colombo and Kandy study scenarios. And, fig 13 depicts the summarized waiting time we have got during both regional scenarios when rerouting was enabled and disabled. 


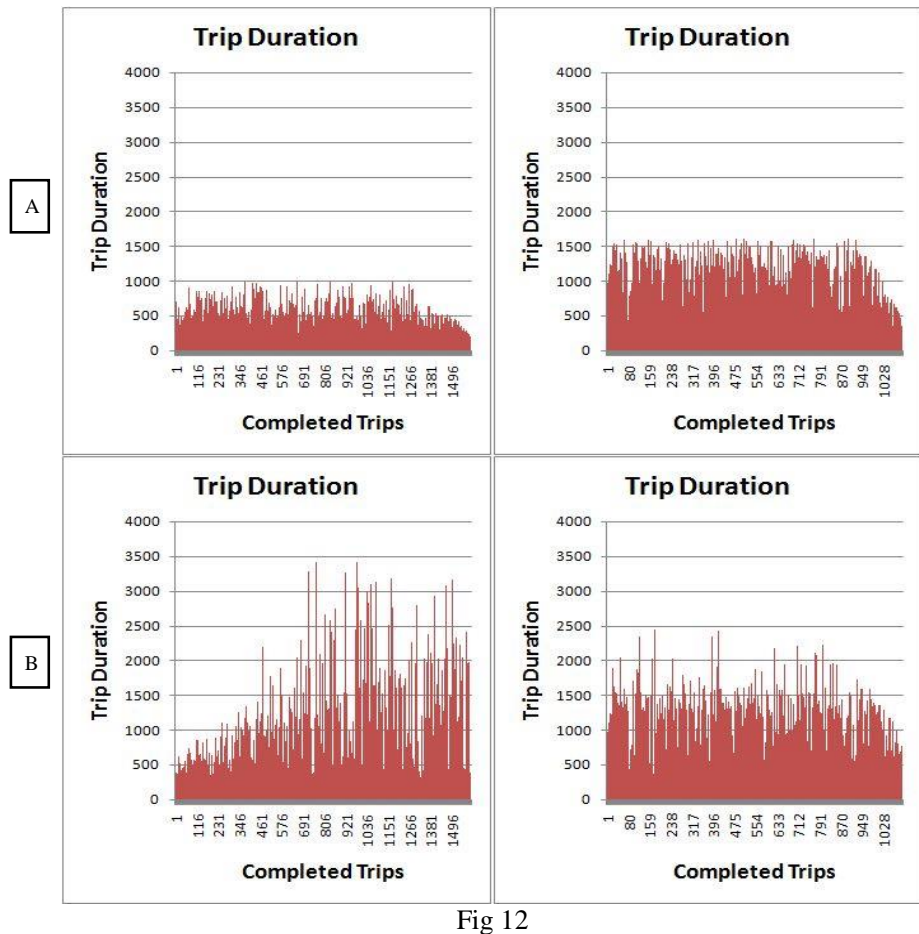

A - Depicts the vehicles trip duration when rerouting approach enabled during Colombo and Kandy scenarios respectively.

B - Depicts the vehicles trip duration when rerouting approach disabled during Colombo and Kandy scenarios respectively.
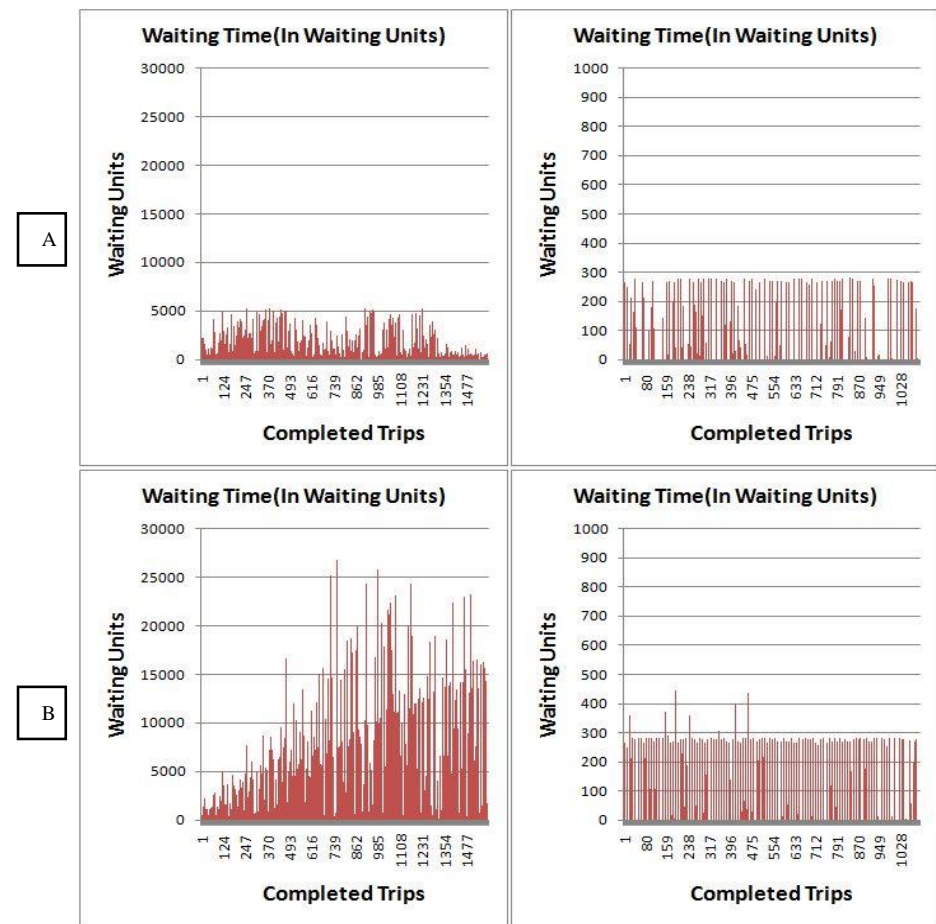

Fig 13

A - Depicts the vehicles waiting time duration when rerouting approach enabled during Colombo and Kandy scenarios respectively.

B - Depicts the vehicles waiting time duration when rerouting approach disabled during Colombo and Kandy scenarios respectively.

Fig 14 and fig 15 visually demonstrates the general vehicle flow in selected intersections from both regional scenarios during the simulations. The screen shots were taken during the identical simulation step when rerouting was enabled and disabled under the two study regions. Other than enabling and disabling the rerouting option all the other conditions and parameters have been kept identical across the simulations to ensure consistency.

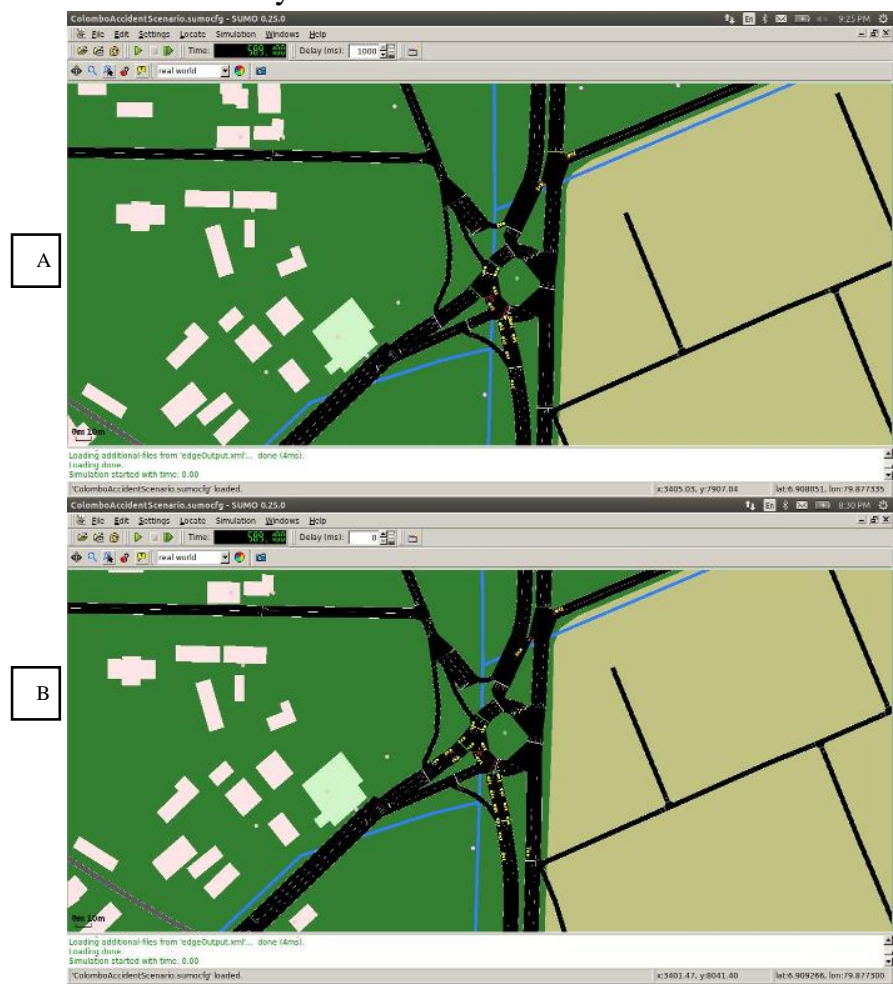

Fig 14 - A, B depicts the vehicle traffic flow near Baudhaloka Mawatha, when rerouting approach enabled and disabled respectively during the Colombo scenario simulation.
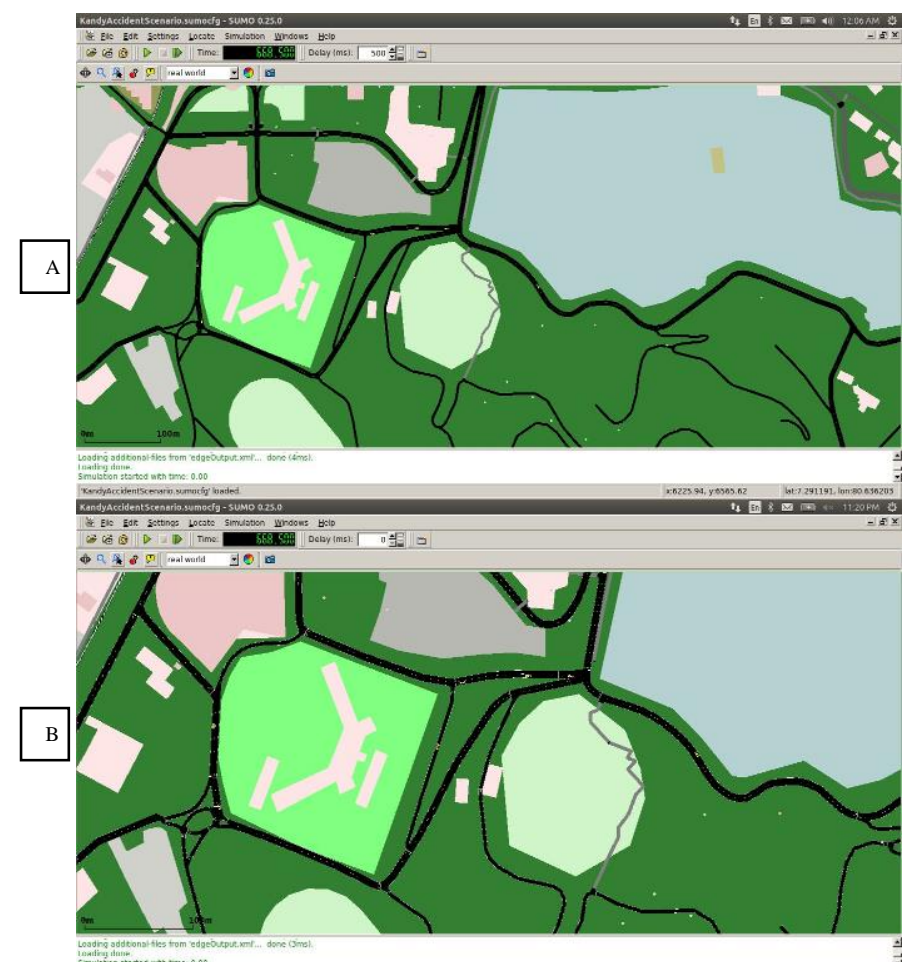

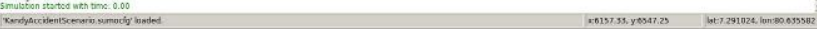
Fig 15 - A, B depicts the vehicle traffic flow near Ehelapola Kumarihami Mawatha, when rerouting approach enabled and disabled respectively during the Kandy scenario simulation.

When we analyse the graphs depicted in fig12 and fig13, it is evident that quantitatively the trip duration and waiting time were significantly reduced when rerouting approach was used during both regional experiments. Also the graphs 
display uneven outcomes with frequent spikes when rerouting was disabled, but more normalized patterns when the rerouting approach was used. Further, when analysing the screenshots illustrated in fig 14 and fig 15, we can observe that the vehicle flow tends to be more even when the rerouting approach was used during the similar simulation steps. Hence, we can also propose that a qualitative improvement exists when using the rerouting approach during the experiments.

\section{RELATED WORK}

Vehicular ad-hoc networks (VANETs) research area is one of the extensively studied fields of research around the world. In this section, we start our focus with the discussion of related research works on a number of popular represented VANET based approaches used for vehicular re-routing discussing about their strengths and weaknesses and later discuss about few notable general traffic solutions comparing with our solution. The work of Zardosht et al [19] is some extent relative to the problem described in our paper, but their focus and outcome differs significantly. The authors have proposed a cooperative collision warning component using VANET which includes a re-routing module to enable rerouting for vehicles when an accident occurs. Their work was impressive and had been a motivation factor for our study. The weakness we found on this work is the too limited evaluation criteria. They have evaluated their solution with 100 vehicles travelling in and only one accident scheduled during their simulation. Biswas et al [20] proposed a model for a collision avoidance system using dedicated short range communication (DSRC) protocol. In this paper, as a precondition the authors assume all the vehicles are equipped with communication devices and they use warning messages passed between them to avoid collision using DSRC protocol. Even the focus of this paper differs significantly from our paper the analysis of communication methods used in this paper was very helpful for us to better learn various vehicular communication protocols. The work of Yang et al [21] Papadimitratos et al [22] and Elbatt et al [23] are the other notable works which have been the useful resources to get ideas about different communication strategies which may be specifically used for implementing VANET solutions in vehicular domain.

Claes et al [24] is another research work which has been an influential factor in our study. In this paper the authors propose an anticipatory vehicle routing solution based on multi-agent systems [25] concepts. The authors evaluate their proposal using a microscopic simulation approach using a comparatively larger traffic region and approximately 25000 vehicles circulating. This paper conceptually differs from our work since it is proposing a general anticipatory routing solution for vehicles, while our paper focus on vehicular rerouting problem after road blocks occur. The concepts and ideas presented in the works of Krajzewicz [26], Behrisch et al [27] and Bieker et al [28] aided us immensely in our study. These papers extensively discuss microscopic vehicular traffic simulation concepts considering larger traffic regions with significant number of vehicles circulating. Also in these papers, the authors specifically targeted the SUMO tool in their use-case evaluations. Since we were also using SUMO simulator as our vehicular traffic simulation platform, these works supported us well. The ideas detailed in the works of
Steinbach et al [29], Varga et al [30] and Colesanti et al [31] in the area of network simulation were among the inspiring thoughts which motivated us during our study. In these papers, the authors explain the concepts of network simulation, ad-hoc wireless networks and possible intervehicle networking architectural patterns using OMNeT++ discrete event simulation tool. Since we have used OMNeT++ as the network simulation platform in our simulations, these research works were extensively helpful in understanding the concepts. In our study we are coupling network simulation and vehicular traffic simulation using an intermediary interface named Veins framework and we are controlling the re-routing decisions through Veins and clearly segregating the networking layer and traffic simulation layer. The research works presented in the papers of Sommer et al [32], Arellano et al [33] and Noori [34] became very useful during our study, since these papers significantly explain the concepts of pairing network simulation and traffic simulation via an intermediary platform and the features available in the Veins framework for this purpose.

Apart from these represented research papers stated above, we feel it is worth to mention few more research works which were helpful for us to understand the general traffic solution concepts. To understand the general concepts of macroscopic, microscopic and mesoscopic simulations, the works of Delis et al [35], Leclercq et al [36], Xiong et al [37], Hermanns et al [38], Balasha et al [39] and Lu et al [40] have been the good reference materials during our study. In these papers the concepts of traffic simulation are well explained according to their purpose and simulation type. The research works of Rakkesh et al [41], Suzumura et al [42] and Wall et al [43] were useful resources to understand the ideas of multi-modal traffic simulation. In these papers, the authors consider the multi-modality of an urban traffic environment when modelling their use-case scenarios. Also the works of Kargl et al [44], Sharef et al [45] and Wong et al [46] in the field of vehicular communication topologies were really useful for us during our study. In these papers, the authors discuss various communication standards and protocols generally used for inter vehicular communication (IVC) interfaces. At last, but not the least the general ideas and concepts detailed in the works of Rakkesh et al [47], Doniec et al [48], Pursula et al [49] and Codeca et al [50] have been the noted inspiration factors for our study. In these papers, the authors present the ideas of how to model the metropolitan traffic scenarios in a simulator environment, define traffic light cycles and phase durations, assign traffic demand, collect the generated output results for analysis, present the results in graphical representations, etc.

\section{CONCLUSIONS}

In this paper, we have proposed an equilibrating strategy which can be implemented as part of a VANET solution, in order to reroute vehicles efficiently when an accident or roadblock occurs in the traffic region. For experimental purposes, we have selected sample study regions from Colombo and Kandy districts in Sri Lanka. The solution we have designed confirms the VANET design requirement and for evaluation purposes we have used the simulation approach to validate our experiments. We have used OMNeT++ as network simulator, SUMO microscopic simulator for traffic simulation and Veins framework as the 
mediating interface between SUMO and OMNeT++. We have performed our trials for both study regions setting rerouting component enabled and disabled as the varying condition while all the other attributes similar during simulations. To analyze the output results, we have selected the trip duration and waiting time of vehicles as quantitative comparison parameters and even vehicle flow as the qualitative comparison metrics. With the results we found out that the trip duration and waiting time of vehicles significantly gets reduced when the rerouting strategy was enabled in the solutions. Also, when rerouting approach was enabled the general traffic flow of vehicles tends to get smoother and more balanced. Hence we can propose that the VANET solution demonstrated in this paper may be used to build an intelligent traffic solution to equipoise an urban traffic scenario, which can better handle road block situations via the proposed rerouting strategy reducing the congestion in the traffic region. With the current solution, we did not consider railway line traffic scenarios, cargo vehicle operations, pedestrian movements and water channel travel options. In the future versions of the solution, we are planning to address these deficits and enhance the solution.

\section{ACKNOWLEDGMENT}

We greatly appreciate and thank our colleagues for their invaluable assistance and constructive feedback on the course of this research study. They have encouraged us significantly during our course work.

\section{REFERENCES}

[1] Alam Muhammad et al., "Introduction to Intelligent Transportation Systems", Intelligent Transportation Systems. Springer International Publishing, 2016.

[2] Martínez Torres et al., "The moderating role of prior experience in technological acceptance models for ubiquitous computing services in urban environments", Technological Forecasting and Social Change 91, 2015, pp. 146-160.

[3] Dar et al., "Wireless communication technologies for ITS applications [topics in automotive networking]", Communications Magazine, IEEE 48.5, 2010, pp. 156-162.

[4] S. Dharmaraja, Resham Vinayak and Kishor S. Trivedi, "Reliability and survivability of vehicular ad hoc networks: An analytical approach", Reliability Engineering \& System Safety 153, 2016, pp. 2838.

[5] Valente and Antonio Saverio, "Cooperative Driving in Inter-Vehicular Communication Network", 2015.

[6] D. Krajzewicz et al, "The open source traffic simulation package SUMO", RoboCup Infrastructure Simulation Competition, 2006.

[7] Varga and Andras, "OMNeT++ - Modeling and Tools for Network Simulation”, Springer Berlin Heidelberg, 2010, pp. 35-59.

[8] Christoph Sommer, Reinhard German and Falko Dressler, "Bidirectionally Coupled Network and Road Traffic Simulation for Improved IVC Analysis", IEEE Transactions on Mobile Computing, vol. 10 (1), 2011, pp. 3-15.

[9] Fernandez et al, "Performance of the $802.11 \mathrm{p}$ physical layer in vehicle-to-vehicle environments", Vehicular Technology - IEEE Transactions on 61.1, 2012, pp. 3-14.

[10] Gozálvez et al., "IEEE 802.11p vehicle to infrastructure communications in urban environments", Communications Magazine IEEE 50.5, 2012, pp. 176-183.

[11] Ghafoor, Kayhan Zrar, et al., "A fuzzy logic approach to beaconing for vehicular ad hoc networks", Telecommunication Systems 52.1, 2013, pp. 139-149.

[12] Abdrabou et al., "Probabilistic delay control and road side unit placement for vehicular ad hoc networks with disrupted connectivity", Selected Areas in Communications - IEEE Journal on 29.1, 2011, pp 129-139.

[13] NS3 Vanet Projects (2016). Ns3 Vanet Simulation Projects. [Online]. Available: http://ns3-code.com/ns3-vanet-projects/
[14] Lu and Hongsheng, "Improving the scalability performance of dedicated short range communications", UNIVERSITY OF NOTRE DAME, 2015.

[15] Phunchongharn, Ekram Hossain, Dong In Kim, et al, "Resource allocation for device-to-device communications underlaying LTEadvanced networks.", Wireless Communications - IEEE 20.4, 2013, pp. 91-100.

[16] M. Haklay and P. Weber, "OpenStreetMap: User-Generated Street Maps", IEEE Pervasive Computing - vol. 7, 2008, pp. 12-18.

[17] Balmer Michael, Kay Axhausen, et al., "Agent based demand modeling framework for large scale micro simulations", Transportation Research Record: Journal of the Transportation Research Board, 2006, pp. 125-134.

[18] Wegener Axel, et al., "TraCI: an interface for coupling road traffic and network simulators", Proceedings of the 11th communications and networking simulation symposium. ACM, 2008.

[19] Zardosht, Besat, Steven Beauchemin, and Michael A. Bauer. "A decision making module for cooperative collision warning systems using Vehicular Ad-Hoc Networks." 16th International IEEE Conference on Intelligent Transportation Systems (ITSC 2013). IEEE, 2013.

[20] Biswas, Subir, Raymond Tatchikou, and Francois Dion. "Vehicle-tovehicle wireless communication protocols for enhancing highway traffic safety." IEEE communications magazine 44.1 (2006): 74-82.

[21] Yang, Xue, et al. "A vehicle-to-vehicle communication protocol for cooperative collision warning." Mobile and Ubiquitous Systems: Networking and Services, 2004. MOBIQUITOUS 2004. The First Annual International Conference on. IEEE, 2004.

[22] Papadimitratos, Panos, et al. "Vehicular communication systems: Enabling technologies, applications, and future outlook on intelligent transportation." IEEE Communications Magazine 47.11 (2009): 8495.

[23] ElBatt, Tamer, et al. "Cooperative collision warning using dedicated short range wireless communications." Proceedings of the 3rd international workshop on Vehicular ad hoc networks. ACM, 2006.

[24] Claes, Rutger, Tom Holvoet, and Danny Weyns. "A decentralized approach for anticipatory vehicle routing using delegate multiagent systems." IEEE Transactions on Intelligent Transportation Systems 12.2 (2011): 364-373.

[25] Ferber, Jacques. Multi-agent systems: an introduction to distributed artificial intelligence. Vol. 1. Reading: Addison-Wesley, 1999.

[26] Krajzewicz, Daniel. "Traffic simulation with SUMO-simulation of urban mobility." Fundamentals of traffic simulation. Springer New York, 2010. 269-293.

[27] Behrisch, Michael, et al. "SUMO-simulation of urban mobility: an overview." Proceedings of SIMUL 2011, The Third International Conference on Advances in System Simulation. ThinkMind, 2011.

[28] Bieker, Laura, et al. "Traffic simulation for all: a real world traffic scenario from the city of Bologna." Modeling Mobility with Open Data. Springer International Publishing, 2015. 47-60.

[29] Steinbach, Till, et al. "Extending OMNeT++ Towards a Platform for the Design of Future In-Vehicle Network Architectures." arXiv preprint arXiv:1609.05179 (2016).

[30] Varga, András, and Rudolf Hornig. "An overview of the OMNeT++ simulation environment." Proceedings of the 1st international conference on Simulation tools and techniques for communications, networks and systems \& workshops. ICST (Institute for Computer Sciences, Social-Informatics and Telecommunications Engineering), 2008.

[31] Colesanti, Ugo Maria, Carlo Crociani, and Andrea Vitaletti. "On the accuracy of omnet++ in the wireless sensornetworks domain: simulation vs. testbed." Proceedings of the 4th ACM workshop on Performance evaluation of wireless ad hoc, sensor, and ubiquitous networks. ACM, 2007.

[32] Sommer, Christoph, Reinhard German, and Falko Dressler. "Bidirectionally coupled network and road traffic simulation for improved IVC analysis." IEEE Transactions on Mobile Computing 10.1 (2011): 3-15

[33] Arellano, Wilmer, and Imad Mahgoub. "TrafficModeler extensions: A case for rapid VANET simulation using, OMNET++, SUMO, and VEINS." 2013 High Capacity Optical Networks and Emerging/Enabling Technologies. IEEE, 2013.

[34] Noori, Hamed. "Realistic urban traffic simulation as vehicular ad-hoc network (vanet) via veins framework." 12th Conference of Open Innovations Framework Prgramm. FRUCT. 2012.

[35] Delis, Anargiros I., Ioannis K. Nikolos, and Markos Papageorgiou. "Macroscopic Modelling and Simulation of Multi-lane Traffic." 2015 IEEE 18th International Conference on Intelligent Transportation Systems. IEEE, 2015. 
[36] Leclercq, Ludovic, et al. "Macroscopic traffic dynamics with heterogeneous route patterns." Transportation Research Part C: Emerging Technologies 59 (2015): 292-307.

[37] Xiong, Chenfeng, et al. "Developing a 24-hour large-scale microscopic traffic simulation model for the before-and-after study of a new tolled freeway in the Washington, DC-Baltimore Region." Journal of Transportation Engineering 141.6 (2015): 05015001.

[38] Hermanns, Gerhard, et al. "Microscopic Simulations of Oversaturated City Traffic: Features of Synchronized Flow Patterns." Traffic and Granular Flow'15 (2015): 35.

[39] Balasha, Tamir, and Tomer Toledo. "MESCOP: A Mesoscopic Traffic Simulation Model to Evaluate and Optimize Signal Control Plans." Transportation Research Record: Journal of the Transportation Research Board 2488 (2015): 1-9.

[40] Lu, Shou-feng, et al. "Mesoscopic Traffic Flow Model Considering Overtaking Requirements." Journal of Highway and Transportation Research and Development (English Edition) 9.4 (2015): 85-90.

[41] Rakkesh, S. T., A. R. Weerasinghe, and R. A. C. Ranasinghe. "Effective urban transport planning using multi-modal traffic simulations approach." 2016 Moratuwa Engineering Research Conference (MERCon). IEEE, 2016.

[42] Suzumura, Toyotaro, Gavin McArdle, and Hiroki Kanezashi. "A high performance multi-modal traffic simulation platform and its case study with the dublin city." Proceedings of the 2015 Winter Simulation Conference. IEEE Press, 2015.

[43] Wall, Thomas A., et al. "A federated simulation method for multimodal transportation systems: combining a discrete event-based logistics simulator and a discrete time-step-based traffic microsimulator." Simulation 91.2 (2015): 148-163.

[44] Kargl, Frank, et al. "Secure vehicular communication systems: implementation, performance, and research challenges." IEEE Communications Magazine 46.11 (2008): 110-118.

[45] Sharef, Baraa T., Raed A. Alsaqour, and Mahamod Ismail. "Vehicular communication ad hoc routing protocols: A survey." Journal of network and computer applications 40 (2014): 363-396.

[46] Wong, K. Daniel, et al. "Inter-Vehicular Communications." IEEE Wireless Commun. 13.5 (2006): 6-7.

[47] Rakkesh, S. T., A. Ruvan Weerasinghe, and RA Chaminda Ranasinghe. "Traffic light optimization solutions using multimodal, distributed and adaptive approaches." Advances in ICT for Emerging Regions (ICTer), 2015 Fifteenth International Conference on. IEEE, 2015.

[48] Doniec, Arnaud, et al. "A behavioral multi-agent model for road traffic simulation." Engineering Applications of Artificial Intelligence 21.8 (2008): 1443-1454.

[49] Pursula, Matti. "Simulation of traffic systems-an overview." Journal of Geographic Information and Decision Analysis 3.1 (1999): 1-8.

[50] Codeca, Lara, et al. "Luxembourg SUMO Traffic (LuST) Scenario: Traffic Demand Evaluation." IEEE Intelligent Transportation Systems Magazine (2016). 